\title{
Predictors of Spontaneous Remodelling of Angular Knee Deformities in Children with Healed Nutritional Rickets: A Prospective Cohort Study Protocol
}

\author{
Dostafa M. Baraka ${ }^{1+}$ (D) Shady Samir' ${ }^{1}$ (D) Shady Mahmoud ${ }^{1}$, (D) Heba Elsedfy', (D) Rana A. \\ Mahmoud $^{2}$, (D) Tamer A. El-Sobky ${ }^{1}$ * \\ ${ }^{1}$ Division of Pediatric Orthopedics, Department of Orthopedic Surgery, ${ }^{2}$ Department of Pediatrics, \\ Faculty of Medicine, Ain Shams University, Cairo, Egypt. \\ +mostafa.baraka@med.asu.edu.eg or*tamer.ahmed@med.asu.edu.eg
}

Keywords: Child nutrition disorders; Vitamin D deficiency rickets; Metabolic bone diseases; Genu valgum; Genu varum; Bone remodelling.

\begin{abstract}
Background: Nutritional rickets is still considered as a global health problem especially in low-resource countries and immigrant societies of developed countries. Generalized skeletal deformities including angular knee deformities as genu varum and genu valgum are prominent features of nutritional rickets. Angular knee deformities can cause gait difficulties. However, the true remodelling potential of the healed knee deformities is largely unknown to the pediatric and orthopedic community. Aims: The primary objective of this study protocol is to investigate the natural history of disease in regard to the remodelling potential of angular knee deformities in children and adolescents with healed nutritional rickets. And determine the potential patient- and deformity-related factors that are associated with a favorable deformity remodelling as age and sex of patients and type, severity, laterality of deformities etc.

Methods: This will be a prospective observational analytical cohort natural history of disease study. A minimum of at least 140 knees with healed nutritional rickets and angular knee deformities will be observed at regular three-monthly intervals over a period of at least one year. We will report two outcome variables namely; clinical and radiological tibio-femoral angle. And independent patient and deformity-related variables as age, sex, deformity type, severity and laterality. Inferential statistics namely bivariate and multivariate analysis will be employed accordingly to identify the relation between outcome variables and the different independent variables.

Ethics and dissemination: This protocol study has been approved by the institutional research ethics committee. Results will be presented at conferences and submitted to relevant international and society publications. Authors will also communicate the results to their scholarly networks and post them on scholarly networking sites.

Conclusions: This study has the potential for improving the decision-making process as to which patients deserve conservative treatment and which deserve surgical deformity correction. Results are expected to have an impact on the clinical practice of pediatricians, orthopedic surgeons and family physicians alike.
\end{abstract}

\section{What is Already Known on this Topic?}

- Angular knee deformities in children with "active" nutritional rickets (NR) have a definite tendency for healing over time either spontaneously or by means of medical treatment.

- Broadly speaking, residual angular knee deformities in children with "healed" NR have a tendency towards spontaneous remodelling.

- The indications of surgical intervention as guided growth to correct healed angular knee deformities in children with NR is not exactly delineated. 


\section{What this Study Hopes to Add?}

- An estimate of the percentage of failures of spontaneous remodelling of residual angular knee deformities in children with healed NR.

- Identify which patient and deformity characteristics are at risk of failing to remodel spontaneously.

- A practice changing evidence-based treatment algorithm to clearly identify the indications of surgical deformity correction.

\section{Introduction}

\subsection{Background and significance}

Nutritional rickets (NR) is still considered as a global health issue especially in low-resource countries spanning vast regions of Asia, Africa and Latin America [1-4]. Paradoxically, even in western countries the vast majority of children with nutritional rickets were immigrants from low-resource countries $[5,6]$. Severe rachitic lower limb deformities as angular knee deformities -genu varum and genu valgum- can be functionally incapacitating and unsightly. Most active bone lesions in children with NR tend to heal spontaneously with or without medical treatment [7]. Evidence from numerous unpublished data and less published data suggest that a considerable percentage of limb deformities in children with healed NR tend to remodel over time [8]. Nevertheless, the predictors of good prognosis in terms of patient- and deformity-related variables are largely unknown. In other words, the patient and deformity characteristics that are associated with a favorable response namely spontaneous deformity remodelling are not clearly specified in the literature [8]. This is because the main bulk of publications on children with NR address prevention [9,10], non-skeletal presenting manifestations [11], medical treatment [7, 12, 13], underlying patho-aetiological factors [14], genetic susceptibility [15], identification of potential risk factors [16], orthopedic surgery management [17], animal experimentation [18] and epidemiological and public health aspects of the disease [19-21]. Further, one publication only addresses the natural history of orthopedic deformities in healed NR and its relation to the surgical decision-making principles [8].

Relatedly, the remodelling potential of malunited lower extremity fractures in healthy growing children is variably influenced by age, deformity severity in the various planes and deformity location among others [22]. However, the complex interplay between these co-variables can cause decision-making challenges as to which patients deserve conservative treatment and which deserve a form of surgical intervention [22, 23]. Likewise, there is considerable evidence to suggest that independent co-variables as age, deformity type, severity and duration, and laterality impact spontaneous deformity correction or remodelling potential of angular knee deformities in healed rachitic children. And it is not exactly known how the interplay between these variables impacts spontaneous deformity correction. Nor is it exactly known what the relative weight of each variable is in influencing spontaneous deformity correction.

This creates surgical decision-making dilemmas with respect to the appropriate indications for intervention by guided growth surgery, a procedure which has demonstrated safety and efficacy in the treatment of childhood angular knee deformities of various etiologies including NR [17, 24]. Guided growth has also demonstrated efficacy in treatment of torsional long bone deformities in clinical [17] and experimental settings [25]. Additionally, these decision-making challenges call for the execution of natural history-of-disease studies to elucidate disease/deformity remodelling or behavior over time. And to investigate the potential patient and deformity co-factors impacting disease remodelling. Recently, a similar study design settled the controversy over the remodelling potential of late valgus deformity secondary to proximal tibial metaphyseal fractures in children, referred to as Cozen's phenomenon [26]. These findings have the potential to facilitate decision-making and avoid unnecessary surgery. We postulate that isolation of predictors of good prognosis and complete deformity remodelling in children with healed NR can help orthopedic surgeons and pediatricians alike to arrive at better informed decisions. Uncovering the true prognosis of such deformities can allow for better medical counselling for parents of children with healed NR.

\subsection{Objectives}

The primary objective of this study protocol is to investigate the natural history of disease in regard to the remodelling potential of angular knee deformities in children and adolescents with healed NR. The secondary objective is to determine the potential 
patient- and deformity-related characteristics that are associated with a favorable deformity remodelling namely age and sex of patients and type, severity, laterality and duration of deformities among others.

\subsection{Study design}

This will be a prospective observational analytical prevalent cohort study [27]. The aim of which is to study the natural history of disease in regard to the remodelling potential of angular knee deformities in children with healed nutritional rickets. Patients will be enrolled once the protocol is approved by the institutional research ethics committee. We will report about children and adolescence with healed nutritional rickets and angular knee deformities -genu valgum and genu varum- in regard to patient demographics as age and sex etc. and deformity characteristics as type, severity, laterality and duration prior to presentation. Patients will be followed up for at least one year from the date of enrollment.

\section{Methods and analysis}

\section{$2.1 \quad$ Study settings}

This will be a prospective observational analytical prevalent cohort natural history of disease study [27]. Patients with healed NR and angular knee deformities will be observed at regular three-monthly intervals over a period of at least one year. The aim of the regular follow-up visits to assess for the status of healing and detect any emerging radiographic or laboratory signs of rachitic activity. The following clinical and radiological parameters will be recorded and correlated over the follow-up period; (a) patients' age, (b) sex, (c) deformity type (varus versus valgus), (d) deformity severity as per tibio-femoral angle, (e) laterality (unilateral versus bilateral) and (f) degree of internal tibial torsion [Figure 1]. Deformity severity will be measured by means of the clinical and radiological antero-posterior tibio-femoral angle. Both clinical and radiologic recordings will be obtained from standing positions with extended knees and neutral rotation of the patella [28]. All outcome measures will be recorded immediate post-enrollment and at the proposed regular follow up intervals. Patients will be followed up for at least one year from the date of enrollment.

The confirmation of the diagnosis of NR will be in accordance with the classic clinical, laboratory and radiologic findings of active nutritional rickets. Healing of rachitic activity will be basically judged according to the serum alkaline phosphatase level in addition to the presence of the typical plain radiographic signs in epiphyses around the knee. Serum $25(\mathrm{OH})$ vitamin D3 will be measured for all patients at the beginning of enrollment and at the final follow up. Parents of patients with healed rickets will be instructed to ensure direct sun exposure of their children of at least an overall period of three hours weekly in the time frame from 10 AM to three PM. And ensure oral supplementation with 25 (oh) vitamin D3 and sufficient intake of calcium-rich diet. Parents will be informed of the importance of prolonged and wide skin exposure to enhance the prophylactic gains.

Patients who do not show signs of clinical deformity improvement within one year of enrollment in spite of maintained healing status will be considered for guided growth surgery as per indications of intervention proposed by El-Sobky et al., 2020 [17]. These patients will be counted as failures of spontaneous deformity remodelling. However, the details and outcomes of the anticipated surgical interventions are beyond the scope of this study.

\subsection{Working definitions}

- Healed rickets: serum alkaline phosphatase and serum parathyroid hormone values above the cutoff (450 IU/L) and (60 $\mathrm{pg} / \mathrm{ml}$ ) respectively will be considered abnormal. Concurrently, patients should have normal radiographs of the knees.

- Varus knee was defined as a clinical and radiologic tibio-femoral angle below $0^{\circ}$. Valgus deviation is counted as a positive angle and varus deviation as negative.

- Valgus knee was defined as a clinical and radiologic tibio-femoral angle above $10^{\circ}$.

- An accepted deformity remodelling was defined as a clinico-radiologic tibio-femoral angle between $0^{\circ}$ and $10^{\circ}$ of valgus or a mechanical axis bisecting the central one third of the widest diameter of the proximal tibial epiphysis [17].

- Complete failure of remodelling was defined as a patient who does not show signs of clinic-radiologic deformity improvement -as per above definition- within one year of enrollment in spite of a maintained healing status.

- Incomplete failure of remodelling was defined as a patient who shows signs of clinic-radiologic deformity improvement but fails to reach the end point of alignment -as per definition- within one year of enrollment in spite of a maintained healing status. 


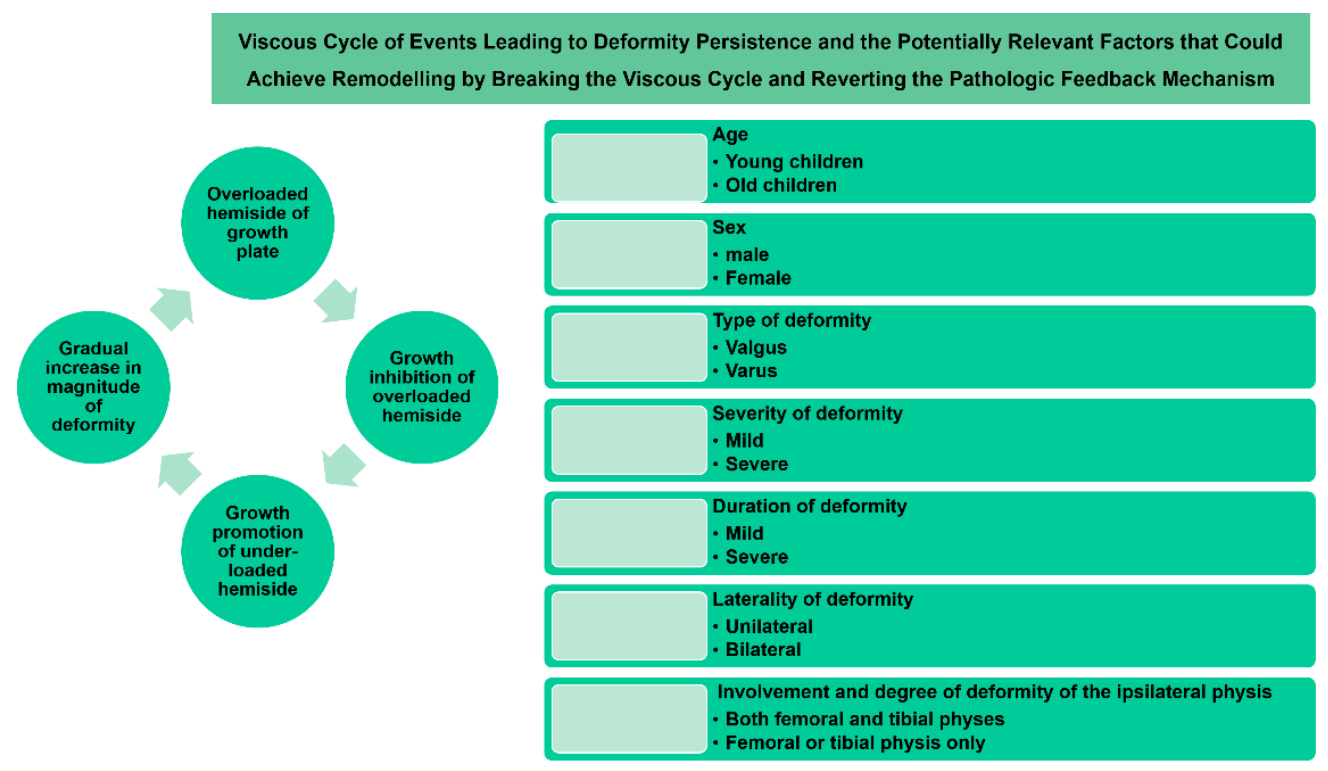

Fig. 1. Potential predictors of remodelling of angular knee deformities in children with healed nutritional rickets.

\subsection{Eligibility criteria}

\subsubsection{Inclusion criteria}

- Children and adolescents aged one year and above with open physes and at least two years of residual physeal growth.

- Healed NR as per definition of healing.

- Unilateral or bilateral coronal plane -angular- deformity around the knee.

\subsubsection{Definitive exclusion criteria}

- Patients under the age of one year old.

- Skeletally mature patients i.e. with a closed physis.

- Patients with active rickets.

- Patients with resistant forms of rickets as genetic and syndromatic or even provisional non-responsiveness to medical treatment.

- Blount disease either in isolation or in association with NR.

- Patients with prior surgical interventions as osteotomies or guided growth.

\subsubsection{Potential exclusion criteria}

- Patients who have been initially included shall be excluded if they demonstrate any laboratory or clinico-radiologic signs of active rickets during the course of follow up.

- Patients who have been initially included but do not show signs of deformity improvement within one year of enrollment i.e. stationary deformity, in spite of maintained healing status. They will be considered for guided growth surgery as per indications of intervention proposed by El-Sobky et al., 2020 [17].

- Patients who have been initially included and show signs of clinic-radiologic deformity improvement but fail to reach the end point alignment -as per definition- within one year of enrollment in spite of a maintained healing status. They will be considered for guided growth surgery as per indications of intervention [17]. Patients who wish to continue conservative management after one year from enrollment despite incomplete deformity correction will not be excluded [Figure 2].

- The above-mentioned patients will be counted as complete and incomplete failures of spontaneous deformity remodelling. However, the details and outcomes of the anticipated surgical interventions are beyond the scope of this study and will not be reported. 
Conservative Treatment Protocol for Angular Knee Deformities in Healed Nutritional Rickets

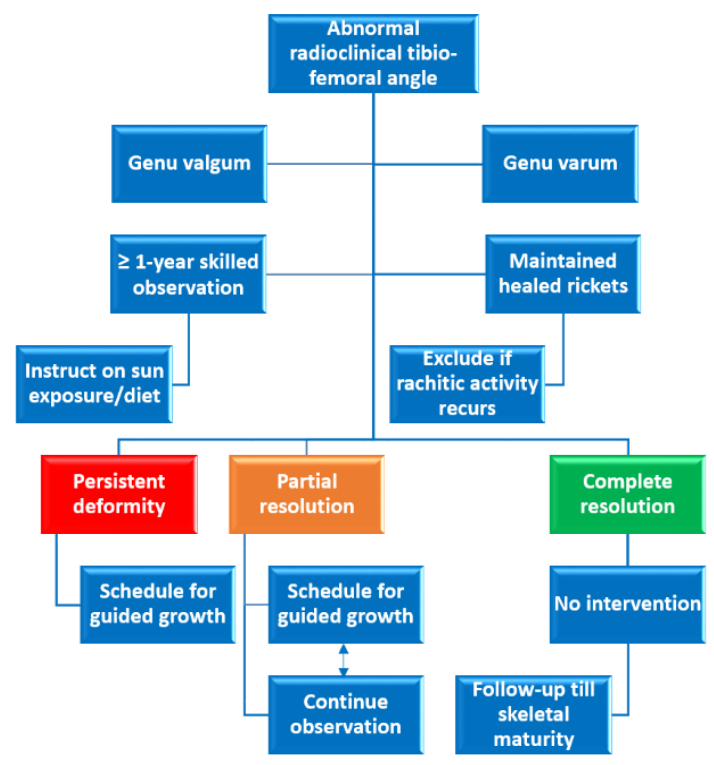

Fig. 2. Conservative treatment protocol for angular knee deformities in healed nutritional rickets.

\subsection{Outcomes}

\subsubsection{Primary objective physician-reported outcome measures}

- The radiologic tibio-femoral angle is created by the intersection of two lines. The first line between centre of proximal diaphysis of femur and centre of distal diaphysis of femur and the second line between centre of proximal diaphysis of tibia and centre of distal diaphysis of tibia.

- The clinical or goniometric tibio-femoral angle is created by the intersection of two lines. The first line joins the anterior superior iliac spine to the centre of the paella and the second line joins the centre of the patella to the centre of the ankle joint i.e. mid-point between both malleoli [28]. Accurate localization of the anterior superior iliac spine entails moving the examiner's fingers across the inguinal ligament proximally and laterally until the first bony prominence is met i.e. from soft tissue to bone.

- The tibial derotation will be measured clinically by the foot-thigh angle in the prone position.

\subsubsection{Secondary subjective patient-reported outcome measures}

- The parents of children with healed NR will be asked to respond to a general and brief questionnaire that is composed of three items namely agility of gait, frequency of falls and satisfaction with cosmetic appearance of lower limbs. The parents will be asked to mark their choices on three-point categorical scale of (a) not satisfied, (b) borderline satisfaction, and (c) fully satisfied.

\subsection{Participant follow-up}

Patients will be seen at regular three-monthly intervals to assess the improvement of angular knee deformity as per described clinico-radiologic measurements. This will depend on the logistic circumstances. Concomitantly, the serum alkaline phosphatase and radiographs of the knees will be assessed to ensure maintained healing status and detect any emerging recurrence of rachitic activity. Parents of will also be consulted in regard to adherence to intake of vitamin D3 supplementations and instructions of life style modification namely maintenance of sufficient and safe sunlight exposure and calcium rich diet as. Unscheduled telephone conversations will be held to further ensure adherence to the named instructions.

\subsection{Sample size and recruitment strategy}


We plan to enroll a convenience sample of at least 140 knees. Sample size was calculated using open-source calculator and based on a study by Prakash et al., [8]. A sample of 140 knees is sufficient to achieve study objectives given that hypothesized $\%$ frequency of outcome factor in the population $(p)$ is $90 \%$; with margin of error $+/-5$, confidence level $95 \%$. We intend to enroll a greater number of patients depending upon availability and logistic issues. This aims at increasing the statistical power of the results. We will analyze data according to both the number of patients and number of knees which is expected to exceed the number of patients due to the possible inclusion of bilateral cases. And we believe that reporting the statistical results as per number of knees is clinically relevant and valid. We will report about children and adolescence with healed NR and angular knee deformities attending the pediatric orthopedic and pediatric endocrinology outpatient clinics at our university hospital.

\subsection{Statistical methods}

Descriptive statistics will be done for all collected variables. Quantitative variables will be presented as minimum, maximum, mean and SD (median and IQR for non-parametric variables). Qualitative variables will be reported as count and percentage. Inferential statistics will be done using the appropriate statistical tests: to find the relation between outcome variables and different independent variables both bivariate and multivariate analysis will be done. Student $t$ test (or Mann Whitney $U$ test for non-parametric variables) will be used for quantitative variables. Chi square test (or Fisher Exact test) will be used for qualitative variables. Logistic regression analysis will be done to measure the independent effect of each variable on outcome. Pearson's or Spearman's correlation coefficient will be used to measure correlation between different continuous quantitative variables. $\mathrm{P}$ value $<$ or $=0.05$ will be considered statistically significant.

\section{Discussion}

There is recognizable basic science knowledge to suggest that mechanical loading of the growth plate has a significant impact on chondrocyte proliferation and hypertrophy and subsequent skeletal genesis [29-32]. These inferences apply to both physiologic and pathologic mechanical stresses. In genu valgum the lateral hemiside of the distal femoral and proximal tibial growth plate are supra-physiologically loaded. Whereas in genu varum the opposite is true. The abnormal or supraphysiological and sustained loading of one hemiside of the growth plate will eventually lead to a differential/unbalanced growth in favour of the relatively unloaded hemiside of the growth plate [Figure 3]. Theoretically, and according to Hueter and Volkmann concept this will create a viscous cycle and lead to persistence or even worsening of any angular knee deformity in children with healed NR at least on the short-term. However, some deformities are able to break this viscous cycle and do remodel over time [8]. These observations suggest that the chondrocyte biological potential in the supra-physiological loaded hemiside of the growth plate responds diversely to joint overloading depending on age, deformity severity, deformity duration, type and laterality in healed NR children.

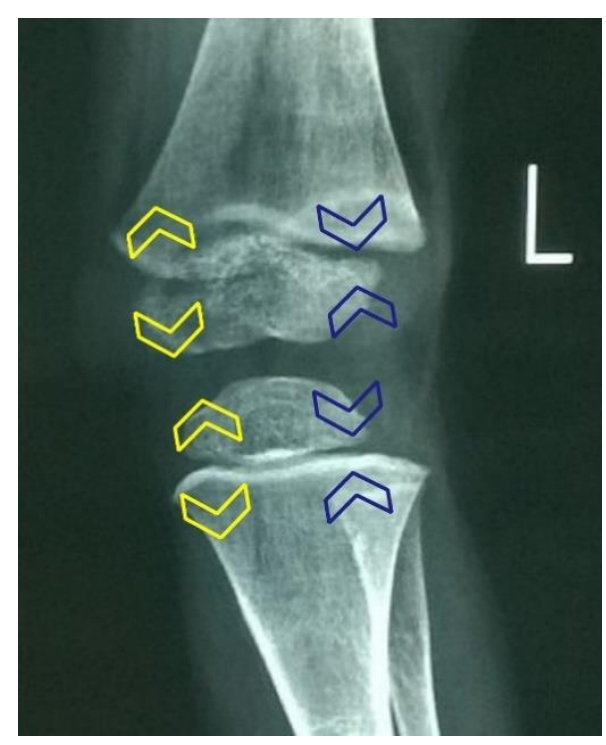

Fig. 3. Proposed pathoaetiology of failure of remodelling in children with healed nutritional rickets. A valgus left knee. Note the compressive forces exerted on the medial hemiside -overloaded- of both the distal femoral and proximal tibial epiphyses (blue arrows). And the tensile forces exerted on the lateral hemiside -underloaded- of the same epiphyses (yellow arrows). 
Generally, the research type and methodological design of the current study protocol has the potential to produce valid results. And the conclusions can be fairly generalized to children with angular knee deformities secondary to healed NR. However, there is diversity in the likely patho-aetiological factors, environmental, socioeconomic, cultural and genetic determinants of disease, and individual responsiveness to medical treatment of children with NR [33-37]. This diversity may also explain the variations in the presenting systemic manifestations -including the orthopedic manifestations- and the clinical course of NR. And it may also have implications for the remodelling potential of healed angular limb deformities either directly or indirectly. These factors have to be taken into account when we interpret the anticipated study conclusions. It would be interesting to explore the possible relationship between the levels of $25(\mathrm{OH})$ vitamin D3.

The final outcomes are expected to serve as a road map for decision-making. This applies to orthopedic surgeons, general pediatricians, pediatric endocrinologists and family physicians. Precision decision-making can potentiate cost-effectiveness and treatment efficiency, and enhance the professionalism of treatment counselling offered to parents of NR children.

\subsection{Ancillary and post-trial care}

The scope of this study protocol does not involve any form of surgical or drug-related intervention. It is anticipated that a considerable portion of the study population will achieve satisfactory deformity remodelling. However, patients who classify as failures at the end of the study will be considered for surgical intervention in the form of guided growth surgery as per indications of intervention [17]. They will be prioritized to receive such treatment when deemed necessary.

Ethics approval: This study was approved by the Medical Ethics Research Committee of Faculty of Medicine, Ain Shams University, Cairo, Egypt, FMASU R 123/2021. Patient consent for publication is not required.

Authors' contributions: TAE conceptualized the research point, designed the protocol and wrote the draft version. MMB participated in the design of the study. All authors participated in critical revision of the draft manuscript and approved the final version.

Appendices: Supplementary file1: Arabic summary available in online version.

\section{References}

[1] Bouillon R, Antonio L. Nutritional rickets: Historic overview and plan for worldwide eradication. J Steroid Biochem Mol Biol. 2020; 198:105563.

[2] Roth DE, Abrams SA, Aloia J, et al. Global prevalence and disease burden of vitamin D deficiency: a roadmap for action in low- and middle-income countries. Ann N Y Acad Sci. 2018; 1430(1):44-79.

[3] Ngari MM, Thitiri J, Mwalekwa L, et al. The impact of rickets on growth and morbidity during recovery among children with complicated severe acute malnutrition in Kenya: A cohort study. Matern Child Nutr. 2018; 14(2):e12569.

[4] Mahmoud AO, Ahmed AY, Aly HM. The prevalence of active nutritional rickets in Egyptian infants in Cairo. Egyptian Pediatric Association Gazette. 2016; 64(3):105-10.

[5] Meyer HE, Skram K, Berge IA, Madar AA, Bjørndalen HJ. Nutritional rickets in Norway: a nationwide register-based cohort study. BMJ Open. 2017; 7(5):e015289.

[6] Thacher TD, Pludowski P, Shaw NJ, Mughal MZ, Munns CF, Högler W. Nutritional rickets in immigrant and refugee children. Public Health Rev. 2016; 37:3.

[7] Lambert AS, Linglart A. Hypocalcaemic and hypophosphatemic rickets. Best Pract Res Clin Endocrinol Metab. 2018; 32(4):455-476.

[8] Prakash J, Mehtani A, Sud A, Reddy BK: Is surgery always indicated in rachitic coronal knee deformities? Our experience in 198 knees. J Orthop Surg (Hong Kong). 2017; 25:2309499017693532.

[9] Gedamu H, Tafere Y. Assessment of knowledge, attitude, and practice of sunlight exposure of infants among mothers attending in governmental health facilities in Farta district, south Gondar zone, north west Ethiopia, 2018. Int J Reprod Med. 2019;2019:2638190.

[10] Kara Elitok G, Bulbul L, Zubarioglu U, et al. How should we give vitamin D supplementation? evaluation of the pediatricians' knowledge in Turkey. Ital J Pediatr. 2017; 43(1):95.

[11] Piloya T, Odongkara B, Were EM, Ameda F, Mworozi E, Laigong P. Nutritional rickets among children admitted with severe pneumonia at Mulago hospital, Uganda: a cross-sectional study. BMC Pediatr. 2018; 18(1):336. 
[12] Chuang LH, Tung YC, Liu SY, Lee CT, Chen HL, Tsai WY. Nutritional rickets in Taiwanese children: Experiences at a single center. $\underline{J}$ Formos Med Assoc. 2018; 117(7):583-587.

[13] Chatterjee D, Swamy MKS, Gupta V, Sharma V, Sharma A, Chatterjee K. Safety and Efficacy of Stosstherapy in Nutritional Rickets. J Clin Res Pediatr Endocrinol. 2017; 9:63-69.

[14] Ahmed S, Goldberg GR, Raqib R, et al. Aetiology of nutritional rickets in rural Bangladeshi children. Bone. 2020; $136: 115357$.

[15] El Kholy M, Elsedfy $H$, Fernández-Cancio M, et al. Nutritional rickets: vitamin D, calcium, and the genetic make-up. Pediatr Res. 2017; 81(2):356-363.

[16] Kassab M, Shaban I, Mohammad K, Creedy DK. Prevalence of hypovitaminosis D among Jordanian healthy infants: a descriptive cross sectional study. J Pediatr Nurs. 2016; 31(2):e119-125.

[17] El-Sobky TA, Samir S, Baraka MM, et al. Growth modulation for knee coronal plane deformities in children with nutritional rickets: A Prospective series with treatment algorithm. J Am Acad Orthop Surg Glob Res Rev. 2020; 4(1):e19.00009.

[18] Dittmer KE, Morley RE, Smith RL. Skeletal deformities associated with nutritional congenital rickets in newborn lambs. $\underline{\mathrm{N} Z \mathrm{Vet} J}$. 2017; 65(1):51-55.

[19] Kubota T, Nakayama $H$, Kitaoka $T$, et al. Incidence rate and characteristics of symptomatic vitamin $D$ deficiency in children: a nationwide survey in Japan. Endocr J. 2018; 65(6):593-599.

[20] Tezera F, Whiting SJ, Gebremedhin S. Dietary calcium intake and sunlight exposure among children aged 6-23 months in Dale Woreda, Southern Ethiopia. Afr. J. Food Agric. Nutr. Dev. 2017; 17(3):12427-12440.

[21] Yasmin, N., Ahmad, S., Sayed, M., Khan, M., \& Karim, M. N. Dietary pattern of rachitic children at selected geographical area. Bangladesh Med J. 2014; 43(3), 151-156.

[22] Stilli S, Magnani M, Lampasi M, Antonioli D, Bettuzzi C, Donzelli O. Remodelling and overgrowth after conservative treatment for femoral and tibial shaft fractures in children. Chir Organi Mov. 2008; 91(1):13-19.

[23] Sanzarello I, Calamoneri E, D'Andrea L, Rosa MA. Algorithm for the management of femoral shaft fractures in children. Musculoskelet Surg. 2014; 98(1):53-60.

[24] Rodrigues NVM, Guarniero R, Boas PJFV, de Miranda BR, Montenegro NB. Hemiepiphysiodesis using eight-plate versus blount staple to correct genu valgum and genu varum. Acta Ortop Bras. 2020; 28(4):195-198.

[25] Cobanoglu M, Cullu E, Kilimci FS, Ocal MK, Yaygingul R. Rotational deformities of the long bones can be corrected with rotationally guided growth during the growth phase. Acta Orthop. 2016; 87(3):301-305.

[26] Papamerkouriou YM, Tsoumpos P, Tagaris G, Christodoulou G. Does Cozen's phenomenon warrant surgical intervention?. J Child Orthop. 2020; 14(3):213-220.

[27] Jewell NP. Natural history of diseases: Statistical designs and issues. Clin Pharmacol Ther. 2016; 100(4):353-361.

[28] Arazi M, Oğün TC, Memik R. Normal development of the tibiofemoral angle in children: a clinical study of 590 normal subjects from 3 to 17 years of age. J Pediatr Orthop. 2001; 21(2):264-267.

[29] Tao F, Jiang T, Tao H, Cao H, Xiang W. Primary cilia: Versatile regulator in cartilage development. Cell Prolif. 2020; 53(3):e12765.

[30] Lee D, Erickson A, Dudley AT, Ryu S. Mechanical stimulation of growth plate chondrocytes: Previous approaches and future directions. Exp Mech. 2019; 59(9):1261-1274.

[31] Moore ER, Jacobs CR. The primary cilium as a signaling nexus for growth plate function and subsequent skeletal development. J Orthop Res. 2018; 36(2):533-545.

[32] Ding J, Jin F, Zhao X, Zhao L, Wu Z, Li J. Hemiepiphysiodesis stapling induces ER stress apoptosis and autophagy in rat growth plates. Am J Transl Res. 2019;11(3):1486-1497.

[33] Moon RJ, Davies JH, Cooper C, Harvey NC. Vitamin D, and Maternal and Child Health. Calcif Tissue Int. 2020; 106(1):30-46.

[34] Al Khalifah R, Alsheikh R, Alnasser Y, et al. The impact of vitamin D food fortification and health outcomes in children: a systematic review and meta-regression. Syst Rev. 2020; 9(1):144.

[35] Ilmiawati C, Oviana A, Friadi A, Reza M. Sunlight exposed body surface area is associated with serum 25-hydroxyvitamin D (25(OH)D) level in pregnant Minangkabau women, Indonesia. BMC Nutr. 2020; 6:18.

[36] Wakayo T, Belachew T, Vatanparast H, Whiting SJ. Vitamin D deficiency and its predictors in a country with thirteen months of sunshine: the case of school children in central Ethiopia. PLoS One. 2015; 10(3):e0120963.

[37] Barry EL, Rees JR, Peacock JL, et al. Genetic variants in CYP2R1, CYP24A1, and VDR modify the efficacy of vitamin D3 supplementation for increasing serum 25-hydroxyvitamin D levels in a randomized controlled trial. J Clin Endocrinol Metab. 2014; 99(10):E2133-E2137. 